\title{
Assimiliert - Abitur, separiert - Hauptschulabschluss? Zum Zusammenhang zwischen Akkulturation und angestrebtem Schulabschluss
}

\author{
Nanine Lilla (i) - Sebastian Thürer • Wim Nieuwenboom • \\ Marianne Schüpbach
}

Eingegangen: 24. März 2020 / Überarbeitet: 18. September 2020 / Angenommen: 22. Februar 2021 /

Online publiziert: 16. März 2021

(C) Der/die Autor(en) 2021

Zusammenfassung Diese Studie geht der Frage nach, inwiefern ein Zusammenhang zwischen der Akkulturation von Schülerinnen und Schülern mit Migrationshintergrund und dem in der Sekundarstufe angestrebten Schulabschluss besteht. Auf Grundlage der Daten des Nationalen Bildungspanels (NEPS) wurden unterschiedliche Aspekte von Akkulturation herangezogen und mittels Latenter Profilanalyse vier Akkulturationsprofile ermittelt: ein assimiliertes, ein moderat assimiliertes, ein integriertes und ein separiertes Akkulturationsprofil. In Abhängigkeit der identifizierten Akkulturationsprofile wurde in logistischen Regressionsanalysen die Chance ein Abitur beziehungsweise einen Hauptschulabschluss anzustreben geschätzt. Vor Berücksichtigung der Deutschkompetenz zeigen die Ergebnisse, dass das separierte Akkulturationsprofil den angestrebten Schulabschluss vorhersagt. Unter Kontrolle der Deutschkompetenz ist für kein Akkulturationsprofil mehr ein Effekt nachweisbar. Darüber hinausgehende Effekte der Herkunftsgruppe können lediglich für die Vorhersage der Chance ein Abitur anzustreben gezeigt werden. Die Befunde reihen sich in den Forschungsstand ein, der auf Nachteile der Separation im deutschen Schulkontext hindeutet und verweisen auf die Bedeutsamkeit der deutschsprachlichen Kompetenzen für schulischen Erfolg.

\footnotetext{
Dr. N. Lilla $(\bowtie) \cdot$ S. Thürer · Dr. W. Nieuwenboom · Prof. Dr. M. Schüpbach

Arbeitsbereich Allgemeine Grundschulpädagogik, Freie Universität Berlin, Habelschwerdter Allee 45, 14195 Berlin, Deutschland

E-Mail: nanine.lilla@fu-berlin.de

S. Thürer

E-Mail: sebastian.thuerer@fu-berlin.de

Dr. W. Nieuwenboom

E-Mail: wim.nieuwenboom@fhnw.ch

Prof. Dr. M. Schüpbach

E-Mail: marianne.schuepbach@fu-berlin.de
} 
Schlüsselwörter Schülerinnen und Schüler mit Migrationshintergrund ·

Akkulturation · Latente Profilanalyse · Bildungsungleichheit

\title{
Assimilation-Abitur, separation-lower school-leaving certificate? The relationship of acculturation and the envisaged school-leaving certificate
}

\begin{abstract}
This study investigates whether there is a relationship between acculturation and the envisaged school-leaving certificate of immigrant students in secondary school. Based on data from the German National Educational Panel Study (NEPS) different aspects of acculturation were employed and four acculturation profiles identified conducting Latent Profile Analysis: an assimilated, a moderate assimilated, an integrated, and a separated acculturation profile. Logistic regression models were run to investigate the odds for envisaging an Abitur or a lower school-leaving certificate dependent on the acculturation profiles identified. Before controlling for linguistic competence, results indicate that the separated acculturation profile predicts the envisaged school-leaving certificate. Controlling for linguistic competence, no acculturation profile shows any effects. Effects of cultural background can only be shown for the predicted odds for envisaging an Abitur. Findings are in line with the state of research, that points to the disadvantages of separation in the German school context and indicate the importance of German language skills regarding academic success.
\end{abstract}

Keywords Acculturation · Educational inequality · Immigrant students · Latent profile analysis

\section{Einleitung}

In Deutschland haben Schülerinnen und Schüler mit Migrationshintergrund vergleichsweise geringere Chancen auf Bildungserfolg. Zuwanderungsbezogene Disparitäten im Kompetenzerwerb zeigen sich bereits in der Primarstufe (z. B. Wendt und Schwippert 2017) und verfestigen sich in der Sekundarstufe (z. B. Weis et al. 2019). Hiesige Forschungsbefunde zum Zusammenhang zwischen der Akkulturation von Schülerinnen und Schülern mit Migrationshintergrund und Schulleistung zeigen jedoch, dass kulturell assimilierte Schülerinnen und Schüler durchaus schulisch erfolgreich sein können (z. B. Edele et al. 2013). Neben dem Kompetenzerwerb stellt die in der Sekundarstufe besuchte Schulform und der damit in Zusammenhang stehende anvisierte Schulabschluss einen wichtigen Indikator für Schulerfolg dar (vgl. Siegert und Olszenka 2016). Vor diesem Hintergrund wird im vorliegenden Beitrag die Verteilung von Schülerinnen und Schülern mit Migrationshintergrund unterschiedlicher Herkunftsgruppen auf verschiedene Bildungsgänge der Sekundarstufe in den Blick genommen und der Zusammenhang zwischen der Akkulturation und dem angestrebten, im Sinne des aufgrund des besuchten Schulzweigs in Aussicht gestellte, Schulabschluss untersucht. Auf Grundlage der Daten der Startkohorte 4 des Nationalen Bildungspanels (NEPS; Blossfeld et al. 2011) werden dazu in einem ersten Schritt mittels Latenter Profilanalyse unterschiedliche Aspekte der Akkultu- 
ration berücksichtigt um vier Akkulturationsprofile zu identifizieren, bevor in einem zweiten Schritt als Kriterium für Bildungserfolg (1) die Chance ein Abitur im Vergleich zu einem niedrigeren Schulabschluss beziehungsweise (2) die Chance einen Hauptschulabschluss im Vergleich zu einem höheren Schulabschluss anzustreben in Abhängigkeit der ermittelten Akkulturationsprofile vorhergesagt wird. Damit zielt diese Studie darauf ab, zum einen Akkulturation valide und in ihrer Komplexität zu erfassen und zum anderen mehr Einsicht in den Zusammenhang von Akkulturation und Bildungserfolg von Schülerinnen und Schülern mit Migrationshintergrund zu erhalten.

\section{Theoretischer und empirischer Hintergrund}

\subsection{Akkulturationsmodell}

Akkulturation bezeichnet allgemein die Prozesse, die ausgelöst werden durch dauerhaften, direkten Kontakt von Individuen unterschiedlicher kultureller Gruppen und Veränderungen auf Seiten einer oder beider Kulturen herbeiführen (Redfield et al. 1936). Einer sozialpsychologischen Auffassung folgend, rekurriert der Kulturbegriff dabei auf gesellschaftlich geteilte Normen, Werte und Überzeugungen sowie Verhaltensweisen und verweist auf Differenzen zwischen gesellschaftlichen Gruppen (Rudmin 2003). Auf Ebene des Individuums wird dieser Prozess auch als psychologische Akkulturation bezeichnet (Graves 1967). Nach Berry (1997) lässt sich die Akkulturation einer Person anhand von zwei Dimensionen charakterisieren: der Orientierung an der Aufnahmekultur und der Orientierung an der Herkunftskultur. Aus der Kombination dieser beiden theoretisch voneinander unabhängigen Dimensionen ergeben sich vier unterschiedliche Akkulturationsmuster: Integration, Assimilation, Separation und Marginalisierung. Integration ist gekennzeichnet durch eine starke Orientierung sowohl an der Aufnahmekultur als auch der Herkunftskultur. Assimilation zeichnet sich aus durch eine starke Orientierung an der Aufnahmekultur und schwache Orientierung an der Herkunftskultur, Separation hingegen durch eine schwache Orientierung an der Aufnahmekultur und starke Orientierung an der Herkunftskultur. Als Marginalisierung wird eine schwache Orientierung an beiden Kulturen bezeichnet.

Diese Unterscheidung von vier Akkulturationsorientierungen liefert nur einen ersten Ordnungsrahmen zur Beschreibung einer sehr viel komplexeren Realität. Berry (1997, 2013) selbst merkt an, dass die Akkulturationsorientierung des Einzelnen nicht unabhängig vom gesellschaftlichen Kontext ist, da die Mehrheitsgesellschaft die Rahmenbedingungen für individuelle Akkulturation setzt. Außerdem haben die Akkulturationserwartungen der larger society einen wesentlichen Einfluss auf die Akkulturationsorientierungen, die Migrantinnen und Migranten offenstehen (Berry 2001, 2006). Diese Minderheitenperspektive, aus der Berrys Akkulturationsmodell heraus entwickelt wurde (vgl. Hachfeld und Syring 2020), steht der Auffassung einer multikulturellen Gesellschaft, in der Mehr- und Minderheiten immer stärker verschwimmen, gegenüber und stellt Berrys Akkulturationsmodell zunehmend in Frage. Das Konzept der Ko-Kulturation (Reinders et al. 2011) geht beispielswei- 
se davon aus, dass gleichberechtigte Aushandlungsprozesse zwischen Mitgliedern unterschiedlicher kultureller Gruppen eher der Lebensrealität von heute in Deutschland aufwachsenden Jugendlichen entspricht als das Verständnis einer Minderheit, die sich in einer Mehrheitsgesellschaft akkulturiert. Unter der Annahme, dass die Idee unterschiedlicher Akkulturationsorientierungen dennoch nicht überholt ist, ist weiter zu beachten, so betont auch Berry (1997), dass die Akkulturationsorientierung eines Individuums durch den situativen Kontext beeinflusst wird und gar je nach Domäne variieren kann (vgl. auch Arends-Tóth und van de Vijver 2004, 2006). So zeigt eine Untersuchung aus den Niederlanden beispielsweise, dass im öffentlichen Bereich zwar die Anpassung an der niederländischen Kultur angestrebt wird, jedoch im privaten Bereich eine starke Orientierung an der Herkunftskultur durch die Pflege von Sprache und Bräuchen vorherrscht (Arends-Tóth und van de Vijver 2004).

Während in der Literatur weitestgehend Einigkeit herrscht im Hinblick auf die bidimensionale Konzeptualisierung von Berrys Akkulturationsmodell, wird gleichzeitig Kritik geübt hinsichtlich der Existenz der vier Akkulturationsmuster (vgl. Nieri et al. 2011; Schwartz und Zamboanga 2008). Als einen Versuch die Validität Berrys Akkulturationsmodell und Existenz der vier Akkulturationsmuster zu prüfen, führten Schwartz und Zamboanga (2008) eine Latente Klassenanalyse auf Basis der Angaben 436 hispanoamerikanischer Studierenden zur Orientierung an der Herkunftskultur und der amerikanischen Kultur durch. Ohne vorab Annahmen über die vorhandenen Muster der Akkulturation zu treffen, wurden sechs Akkulturationsklassen empirisch ermittelt. Davon konnten drei Klassen inhaltlich der Integration und je eine Klasse der Assimilation und der Separation zugeordnet werden. Eine weitere Klasse, welche am ehesten der Marginalisierung ähnelte, wurde als ,undifferentiated" klassifiziert. Mit einem ähnlichem Vorgehen identifizierten Fox et al. (2013) in einer Stichprobe von 227 College-Studierenden unterschiedlicher ethnischer Minderheiten hingegen lediglich drei Akkulturationsprofile: Integration, Assimilation und Separation. Marginalisierung als eigenständiges Akkulturationsprofil konnte in dieser Studie nicht empirisch nachgewiesen werden. Nieri et al. (2011) untersuchten in ihrer Studie mit 1632 mexikanisch-stämmigen Jugendlichen in den USA, inwiefern unterschiedliche Aspekte von Akkulturation zusammen ein Akkulturationskonstrukt bilden und darauf basierend Individuen latenten Klassen zuordnet. Dazu berücksichtigten sie Maße zur Einstellung zur mexikanischen und amerikanischen Kultur, zum Akkulturationsverhalten und Sprachgebrauch, den Generationenstatus, die Aufenthaltsdauer in den USA und den Kontakt mit der Herkunftskultur sowie eine Skala zur ethnischen Identität. Das Ergebnis der Latenten Klassenanalyse ergab, dass die Verteilung der Daten am besten durch fünf Akkulturationsklassen wiedergegeben wird, die in Anlehnung an Berry inhaltlich interpretiert werden können als: Integration in erster Einwanderergeneration, Integration in zweiter Generation, Assimilation, Separation und Marginalisierung.

\subsection{Akkulturation und schulischer Erfolg}

Laut Berry (1997, 2005) stehen die vier Akkulturationsmuster in Zusammenhang mit einer mehr oder weniger erfolgreichen Anpassung an die Erfordernisse des Aufnahmekontexts: Integration stellt das anpassungsfähigste Akkulturationsmuster 
dar, wohingegen Marginalisierung als am wenigsten adaptiv gilt. Assimilation und Separation werden als mittelmäßig für eine erfolgreiche Anpassung an den Aufnahmekontext gesehen. Für Schülerinnen und Schüler mit Migrationshintergrund, die sich in dauerhaftem Kontakt mit der Herkunftskultur ihrer Familie und - unter anderem bedingt durch den Schulbesuch - mit der Kultur des Aufnahmelands befinden, stellt die Bewältigung des schulischen Alltags einen wichtigen Aspekt erfolgreicher Anpassung dar.

In Einklang mit den theoretischen Annahmen zeigen zahlreiche US-amerikanische Studien überwiegend positive Zusammenhänge zwischen Integration und schulischer Anpassung. Es existieren jedoch auch Befunde, die auf Vorteile der Assimilation im Hinblick auf Schulleistung und schulische Anpassung verweisen (Makarova und Birman 2015).

Für den deutschen Kontext spricht die Studienlage ebenfalls für Vorteile der Assimilation. Mit einer Analyse der deutschen Stichprobe von PISA 2009 zeigen Edele et al. (2013), dass assimilierte Jugendliche im Vergleich zu einheimischen Peers höhere Lesekompetenzen aufweisen, während für integrierte Jugendliche kein Unterschied in der Lesekompetenz festgestellt werden kann. Schwächere Lesekompetenzen für separierte Jugendliche können bei zusätzlicher Kontrolle der in der Familie gesprochenen Sprache nicht mehr gegen den Zufall abgesichert werden. Für die Gruppe marginalisierter Jugendlicher zeigen sich signifikant niedrigere Lesekompetenzen im Vergleich mit den einheimischen Peers. Ähnlich diesem Befundmuster zeigen die Ergebnisse einer Studie von Hannover et al. (2013) zum Zusammenhang zwischen dem schulischen Selbstbild von Jugendlichen mit Migrationshintergrund und der Lesekompetenz, dass Schülerinnen und Schüler, deren kulturelle Selbstbeschreibung sowohl Deutschland als auch ihre Herkunft enthielt, keinen Vorteil gegenüber Schülerinnen und Schülern hatten, deren Selbstbild sich ausschließlich auf Deutschland bezog. In einer weiteren Studie mit einer Stichprobe von Jugendlichen in Deutschland zeigt sich, dass ein positiver Zusammenhang zwischen der Orientierung an der Aufnahmekultur und den Kompetenzen in Mathematik und Lesen sowie den Noten in beiden Domänen besteht. Die Orientierung an der Herkunftskultur zeigt hingegen einen negativen Zusammenhang mit Mathematikkompetenz und negative jedoch nicht signifikante Zusammenhänge mit der Lesekompetenz und den Noten in Mathematik und Deutsch. Für eine bi-kulturelle Orientierung, die auf einen Vorteil der Integration verweisen würde, konnte für keine der untersuchten Outcome-Variablen ein signifikanter Zusammenhang gezeigt werden (Schotte et al. 2018).

\subsection{Herkunftsbedingte Disparitäten in der Sekundarstufe}

Neben dem Kompetenzerwerb stellt ab der Sekundarstufe die Bildungsbeteiligung einen Indikator für Bildungserfolg dar (z. B. Baumert und Schümer 2001). In Bezug auf die Bildungsbeteiligung von Schülerinnen und Schülern mit Migrationshintergrund haben Studien zum Übergang in die Sekundarstufe einheitlich gezeigt, dass Schülerinnen und Schüler mit Migrationshintergrund deutlich geringere Chancen haben den Übergang auf eine höhere Schulform als die Hauptschule zu vollziehen. Dabei konnten die ungleichen Chancen vollständig auf die in der Primarschule ge- 
zeigten schulischen Leistungen und die ungünstigere soziale Herkunft zurückgeführt werden (vgl. Dollmann 2016).

Für Schülerinnen und Schüler mit Migrationshintergrund bedeutet das eine nachteilige Situation sowohl im Hinblick auf den weiteren Kompetenzerwerb als auch den voraussichtlich zu erreichenden Schulabschluss. Da sich die Schülerschaft auf Hauptschulen im Vergleich zur Schülerschaft auf Gymnasien insbesondere auszeichnet durch einen hohen Migrationsanteil, eine geringe Lesekompetenz, einen niedrigen soziö̈konomischen Status und elterlichen Bildungsstand (Autorengruppe Bildungsberichterstattung 2018) bietet der Besuch der Hauptschule im Gegensatz zum Gymnasium ein schlechteres Lernklima und einen ungünstigeren Sozialisationskontext (Solga und Wagner 2010). Darüber hinaus stellt der Hauptschulbesuch nur das Erreichen eines Hauptschulabschlusses in Aussicht, während der Besuch eines Gymnasiums mit der Aussicht auf den höchsten schulischen Abschluss, das Abitur, verknüpft ist. Mit der Etablierung neuer Sekundarschulformen mit mehreren Bildungsgängen, die ein längeres gemeinsames Lernen ermöglichen, wurde eine bessere Durchmischung angestrebt um der schulartspezifischen soziokulturellen Segregation entgegenzuwirken (Lenz et al. 2019). Dennoch zeigt die prozentuale Verteilung von Schülerinnen und Schülern mit und ohne Migrationshintergrund auf unterschiedliche Schulzweige der Sekundarstufe in der 9. Klasse das altbekannte Muster: Die Anteile der Schülerinnen und Schüler ohne Migrationshintergrund, die eine Hauptschule oder einen Hauptschulzweig besuchen liegen bei 16,4\% und die ein Gymnasium oder einen gymnasialen Bildungszweig besuchen bei 41,3\%, während Schülerinnen und Schüler mit Migrationshintergrund zu 28,1\% die Hauptschule oder einen Hauptschulzweig und lediglich zu 31,5\% ein Gymnasium oder einen gymnasialen Bildungszweig besuchen. Diese Ungleichverteilung verstärkt sich bei besonderer Betrachtung der beiden größten Herkunftsgruppen: So ist die Hauptschul(zweig)quote von Schülerinnen und Schülern mit Herkunft aus der ehemaligen UdSSR und der Türkei mehr als doppelt so hoch $(34,0 \%$ bzw. 36,4\%) wie für 15-Jährige ohne Migrationshintergrund und nur 25,3\% der Jugendlichen mit Herkunft aus der ehemaligen UdSSR und 22,7\% mit türkischem Migrationshintergrund besuchen ein Gymnasium oder gymnasialen Zweig (Olczyk et al. 2016).

Das besonders nachteilige Abschneiden der Schülerinnen und Schüler mit Herkunft aus der ehemaligen UdSSR und insbesondere der Schülerinnen und Schüler mit türkischem Migrationshintergrund ist im Einklang mit nach Herkunftsgruppen differenzierten Analysen der Bildungsbeteiligung 15-jähriger Schülerinnen und Schüler mit Migrationshintergrund in früheren Studien (vgl. Müller und Stanat 2006; Segeritz et al. 2010). Auch hier war die Hauptschulquote von Schülerinnen und Schülern aus der ehemaligen UdSSR und der Türkei mehr als doppelt so hoch wie für 15-Jährige ohne Migrationshintergrund (Müller und Stanat 2006). Im Hinblick auf die Chance eines Gymnasialbesuchs ließ sich für türkischstämmige Jugendliche und Jugendliche aus der UdSSR im Vergleich zu einheimischen Jugendlichen eine geringere Chance zeigen, die für Jugendliche aus der ehemaligen UdSSR jedoch nach Berücksichtigung beispielweise des elterlichen Bildungsniveaus, nicht mehr signifikant war. Jugendliche mit polnischem Migrationshintergrund hatten nach Berücksichtigung des familiären Hintergrunds sogar höhere Chancen auf einen Gymnasialbesuch als Jugendliche ohne Migrationshintergrund (Segeritz et al. 2010). 


\section{Fragestellungen}

Ausgehend vom Forschungsstand zum Zusammenhang zwischen Akkulturation und Schulerfolg sowie der Ungleichverteilung von Schülerinnen und Schülern mit Migrationshintergrund aus unterschiedlichen Herkunftsgruppen auf verschiedene Schulformen beziehungsweise Bildungsgänge der Sekundarstufe des deutschen Bildungssystems, wird in dieser Studie untersucht, inwiefern die Akkulturation von Schülerinnen und Schülern mit Migrationshintergrund in Zusammenhang mit dem angestrebten Schulabschluss steht. Um der Komplexität des Akkulturationskonstrukts gerecht zu werden, werden im Unterschied zur bislang in Akkulturationsstudien vorherrschenden Konzentration auf nur einzelne Aspekte, z. B. die ethnische Identität (Edele et al. 2013; Hannover et al. 2013) oder Akkulturationseinstellungen (Schotte et al. 2018), in dieser Studie unterschiedliche Aspekte der Akkulturation berücksichtigt. Neben der kulturellen Identität und der Akkulturationseinstellung, werden auch Verhaltensaspekte sowie die sprachliche Akkulturation berücksichtigt. In Anlehnung an die berichteten Studien aus den USA werden so in einem ersten Schritt mittels Latenter Profilanalyse Muster der Akkulturation identifiziert und folgender Fragestellung nachgegangen: 1) Inwiefern lassen sich die vier Akkulturationsmuster sensu Berry (1997) - Integration, Assimilation, Separation und Marginalisierung - mittels Latenter Profilanalyse replizieren?

Weiter werden in einem zweiten Schritt auf Basis der identifizierten Akkulturationsprofile folgende Fragestellungen untersucht: 2a) Inwiefern stehen die Akkulturationsprofile im Zusammenhang mit der Chance ein Abitur versus einen niedrigeren Schulabschluss anzustreben? 2b) Inwiefern steht die Herkunftsgruppe nebst den Akkulturationsprofilen im Zusammenhang mit der Chance ein Abitur versus einen niedrigeren Schulabschluss anzustreben? 3a) Inwiefern stehen die Akkulturationsprofile im Zusammenhang mit der Chance einen Hauptschulabschluss versus einen höheren Schulabschluss anzustreben? 3b) Inwiefern steht die Herkunftsgruppe nebst den Akkulturationsprofilen im Zusammenhang mit der Chance einen Hauptschulabschluss versus einen höheren Schulabschluss anzustreben?

In Anlehnung an den deutschen Forschungsstand wird dabei angenommen, dass sich insbesondere Vorteile der Assimilation und Nachteile der Separation und Marginalisierung im Hinblick auf den angestrebten Schulabschluss zeigen lassen. AuBerdem werden Nachteile insbesondere für Schülerinnen und Schüler türkischer Herkunft und aus der ehemaligen UdSSR Stammende erwartet, während dies für Schülerinnen und Schüler mit polnischem Migrationshintergrund nicht der Fall sein sollte. 


\section{Methoden}

\subsection{Stichprobe}

Grundlage für die Analysen ist der Datensatz der Startkohorte 4 des Nationalen Bildungspanels (NEPS; Blossfeld et al. 2011) ${ }^{1}$. Startkohorte 4 umfasst ein repräsentatives Sample von über 15.000 circa fünfzehnjährigen Schülerinnen und Schülern in der Sekundarstufe (9. Klasse) des deutschen Bildungssystems. Insgesamt liegt bei 5778 Schülerinnen und Schülern die Angabe vor, dass sie selbst oder mindestens ein Eltern- oder Großelternteil in einem anderen Land als Deutschland geboren wurde. Für unsere Analysen wurden nur diejenigen Schülerinnen und Schüler mit Migrationshintergrund berücksichtigt, die den vier zahlenmäßig größten Herkunftsgruppen (Polen: $n=911$; Türkei: $n=899$; ehemalige UdSSR: $n=827$; Nord- und Westeuropa: $n=477)$ zuzuordnen sind.

\subsection{Abhängige Variablen}

Der angestrebte Schulabschluss wurde in Anlehnung an Baier und Pfeiffer (2014) operationalisiert über die in der 9. Klasse besuchte Schulform. Bei Besuch einer Schule mit mehreren Bildungsgängen war die Schulzweigzugehörigkeit ausschlaggebend für die Zuordnung. Keinem Schulzweig eindeutig zuzuordnen waren 23,2\% $(n=722)$. Insgesamt konnten 861 einem Abitur (27,6\%), 601 einem Realschulabschluss $(19,3 \%)$ und 930 Schülerinnen und Schüler einem Hauptschulabschluss $(29,9 \%)$ als angestrebtem Schulabschluss zugeordnet werden.

\subsection{Unabhängige Variablen}

Die kulturelle Identität (Phinney 1992) wurde über je ein Item zur Zugehörigkeit zur Aufnahme- und zur Herkunftsgesellschaft abgefragt (z.B. Und wie sehr fühlen Sie sich den Menschen aus Deutschland insgesamt zugehörig?) und jeweils über eine fünfstufige Antwortskala ( $1=$ gar nicht, $2=$ eher nicht, $3=$ mittelmäßig, $4=$ eher stark und 5= sehr stark) erfasst (Zugehörigkeit zur Aufnahmegesellschaft: $n=1814$, $M=3,70, S D=0,95$; Zugehörigkeit zur Herkunftsgesellschaft: $n=1867, M=3,56$, $S D=1,18)$.

In Anlehnung an Phinney (1992) wurden zur Erfassung der Einstellungskomponente von Akkulturation je vier Items zur Verbundenheit mit der Aufnahmeund Herkunftskultur (z.B. Ich fühle mich eng verbunden mit der deutschen Kultur) auf einer vierstufigen Ratingskala (1=trifft gar nicht zu bis $4=$ trifft völlig $z u$ ) abgefragt (Verbundenheit zur Aufnahmekultur: $n=1840$, Cronbachs $\alpha=0,89$,

\footnotetext{
1 Diese Arbeit nutzt Daten des Nationalen Bildungspanels (NEPS): Startkohorte Klasse 9, https://doi. org/10.5157/NEPS:SC4:10.0.0. Die Daten des NEPS wurden von 2008 bis 2013 als Teil des Rahmenprogramms zur Förderung der empirischen Bildungsforschung erhoben, welches vom Bundesministerium für Bildung und Forschung (BMBF) finanziert wurde. Seit 2014 wird NEPS vom Leibniz-Institut für Bildungsverläufe e. V. (LIfBi) an der Otto-Friedrich-Universität Bamberg in Kooperation mit einem deutschlandweiten Netzwerk weitergeführt.
} 
$M=3,00, S D=0,76$; Verbundenheit zur Herkunftskultur: $n=1753$, Cronbachs $\alpha=0,92, M=3,03, S D=0,86$ ).

Die Verhaltenskomponente von Akkulturation wurde in Anlehnung an Schnell (1990) im Hinblick auf die Ausübung kultureller Gewohnheiten des Herkunftslandes abgefragt (z.B. Wie oft hören sie Musik aus diesem Land?) und konnte auf einer fünfstufigen Skala ( $1=$ nie, $2=$ selten, $3=$ manchmal, $4=o f t, 5=i m m e r)$ beantwortet werden ( $n=1794$, Cronbachs $\alpha=0,73, M=3,17, S D=0,94)$.

Zur Erfassung der sprachlichen Komponente der Akkulturation wurden die Angaben zum Sprachgebrauch mit Mutter, Vater und Geschwistern verwendet (Kenji und D'Andrea 1992). Das Antwortformat sah ein semantisches Differential vor (1=nur Deutsch, 2 = meistens Deutsch, manchmal die andere Sprache, $3=$ meistens die andere Sprache, manchmal Deutsch, $4=$ nur die andere Sprache) $(n=1874$, Cronbachs $\alpha=0,78, M=2,27, S D=0,82$ ).

\subsection{Kontrollvariablen}

Zur Ermittlung des elterlichen Bildungsniveaus wurden die Bildungsabschlüsse der Eltern nach der International Standard Classification of Education (ISCED; OECD 1999) herangezogen und der jeweils höhere Bildungsabschluss ausgewählt (HISCED) ( $n=1467, M=4,99, S D=2,90)$.

Als weitere Kontrollvariable wurde das Geschlecht der Schülerinnen und Schüler berücksichtigt (männlich=0; $n=1529(49,1 \%)$; weiblich = 1; $n=1585(50,9 \%)$ ).

Um die Kompetenzen in Deutsch und in Mathematik zu berücksichtigen wurde auf die im NEPS erfasste Lesekompetenz und mathematische Kompetenz zurückgegriffen und die zur Verfügung gestellten WLE-Schätzer (Weighted Maximum Likelihood Estimates, siehe Pohl und Carstensen 2012) verwendet (Deutsch: $n=2648$, $M=-0,30, S D=1,28$; Mathe: $n=2935, M=-0,23, S D=1,16)$.

\subsection{Statistisches Vorgehen}

Die statistischen Analysen erfolgten mit Stata 15.1. Alle Werte wurden z-transformiert. Um Fragestellung 1) zu untersuchen wurde eine Latente Profilanalyse (LPA) gerechnet. Die LPA eignet sich, um in einer gegebenen Stichprobe homogene Subgruppen, sogenannte latente Profile, auf Basis einer Reihe kategorialer und kontinuierlicher Daten zu ermitteln (Nylund-Gibson und Choi 2018). Im Hinblick auf unsere Analyse wird davon ausgegangen, dass die Akkulturationsmuster innerhalb der Stichprobe als latente Profile vorhanden sind (Nieri et al. 2011). Auf Basis der unterschiedlichen Aspekte von Akkulturation, nämlich ethnische Identität, Akkulturationseinstellung, Akkulturationsverhalten und linguistische Akkulturation, wurden vier Akkulturationsprofile ermittelt und inhaltlich interpretiert.

Zur Untersuchung der weiteren Fragestellungen wurden die ermittelten Akkulturationsprofile als Prädiktoren in logistische Regressionsmodelle zur Vorhersage der Chance ein Abitur anzustreben (Frage 2a/b) bzw. einen Hauptschulabschluss anzustreben (Frage 3a/b) aufgenommen. Personen, die keinem Schulabschluss eindeutig zugeordnet werden konnten, wurden aus den Modellen ausgeschlossen. Die Modelle wurden jeweils schrittweise aufgebaut. Zunächst wurde jeweils der Einfluss der 
Akkulturationsprofile untersucht (Modell 1), dann das Geschlecht des Schülers bzw. der Schülerin und das elterliche Bildungsniveau hinzugefügt (Modell 2), anschlieBend wurden zusätzlich Kompetenzschätzer in Deutsch (Modell 3) und Mathematik berücksichtigt (Modell 4) und noch die Herkunftsgruppe einbezogen (Modell 5). Vor Durchführung der logistischen Regressionen wurden fehlende Werte mittels multipler Imputation geschätzt.

\section{Ergebnisse}

\subsection{Beschreibung der ermittelten Akkulturationsprofile}

Abb. 1 zeigt die vier durch LPA ermittelten Akkulturationsprofile mit den jeweiligen Ausprägungen (z-Werte) auf den Variablen kulturelle Identität, Akkulturationseinstellung, Akkulturationsverhalten und linguistische Akkulturation.

Akkulturationsprofil 1 zeichnet sich aus durch eine über dem Stichprobenmittelwert liegende Zustimmung im Hinblick auf die Zugehörigkeit zur Aufnahmegesellschaft sowie der Verbundenheit zur deutschen Kultur und eine deutlich unter dem Stichprobenmittelwert liegende Zustimmung im Hinblick auf die Zugehörigkeit zur Herkunftsgesellschaft sowie der Verbundenheit zur jeweiligen Herkunftskultur. Die unter dem Stichprobenmittelwert liegenden Ausprägungen im Hinblick auf die kulturellen Gewohnheiten und den Sprachgebrauch innerhalb der Familie lassen weiter auf eine relativ geringe Orientierung an der Herkunftskultur schließen. In Anlehnung

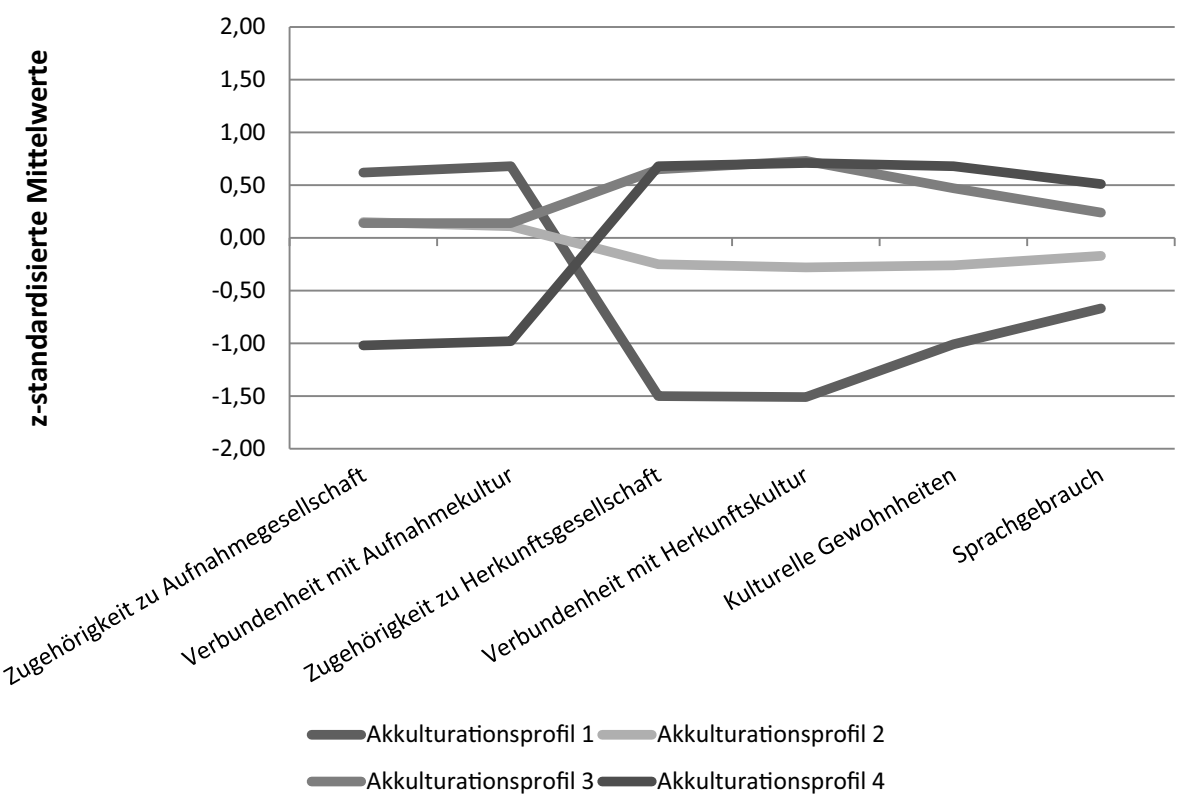

Abb. 1 Graphische Darstellung der Akkulturationsprofile $(N=1348)$ 
an Berry (1997) wird dieses Profil, welches das Muster der individuellen Akkulturation von 8,8\% der Stichprobe widerspiegelt, als assimiliertes Profil bezeichnet.

Akkulturationsprofil 2 und Akkulturationsprofil 3 ähneln sich in der leicht über dem Stichprobenmittelwert liegenden Zustimmung im Hinblick auf die Zugehörigkeit zur Aufnahmegesellschaft sowie der Verbundenheit zur deutschen Kultur, unterscheiden sich jedoch im Muster der mittleren Zustimmung auf den weiteren Variablen. Akkulturationsprofil 2 zeichnet sich aus durch leicht unter dem Stichprobenmittelwert liegende Werte im Hinblick auf die Zugehörigkeit zur Herkunftsgesellschaft, der Verbundenheit zur Herkunftskultur, die kulturellen Gewohnheiten und den Sprachgebrauch innerhalb der Familie. Akkulturationsprofil 3 hingegen zeichnet sich aus durch leicht über dem Stichprobenmittelwert liegende Werte im Hinblick auf die Zugehörigkeit zur Herkunftsgesellschaft, die Verbundenheit zur Herkunftskultur, die kulturellen Gewohnheiten und den Sprachgebrauch innerhalb der Familie. Aufgrund der moderaten Zuwendung zur Kultur des Aufnahmelands bei gleichzeitig moderater Distanz zur Kultur des Herkunftslands, wird Akkulturationsprofil 2, welchem 38,9\% der Stichprobe zugeordnet werden können als moderat assimiliertes Profil bezeichnet. Akkulturationsprofil 3 wird als integriertes Profil bezeichnet, da die Werte darauf hinweisen, dass sich die hierin befindlichen 30,4\% der Schülerinnen und Schüler sowohl an der Kultur des Aufnahme- wie auch des Herkunftslandes orientieren.

Akkulturationsprofil 4 grenzt sich durch negative z-Werte bei der Zugehörigkeit zur Aufnahmegesellschaft und der Verbundenheit zur deutschen Kultur deutlich von den anderen drei Profilen ab. Des Weiteren zeichnet sich dieses Profil durch die positiven z-Werte bei der Zugehörigkeit zum Herkunftsgesellschaft und der Verbundenheit zur Herkunftskultur aus. Auch im Hinblick auf die kulturellen Gewohnheiten und den Sprachgebrauch innerhalb der Familie liegen die Werte über dem Stichprobenmittelwert und können als eine starke Orientierung an der Herkunftskultur interpretiert werden. Da die Zustimmung zur Kultur des Aufnahmelands schwach, aber die Zustimmung zur Kultur des Herkunftslands stark ist, wird dieses Profil, das auf 21,9\% der Stichprobe zurückgeht, in Anlehnung an Berry (1997) als separiertes Profil bezeichnet.

\subsection{Zusammenhang zwischen Akkulturation und der Chance ein Abitur anzustreben}

Anhand von logistischen Regressionsmodellen wurde die Chance ein Abitur versus einen niedrigeren Schulabschluss (Real- und Hauptschulabschluss) anzustreben unter der Voraussetzung unterschiedlicher Akkulturationsprofile (Forschungsfrage 2a) und der Herkunftsgruppe (Forschungsfrage 2b) untersucht. Tab. 1 zeigt die Koeffizienten der logistischen Regressionsanalyse.

In Modell 1 wird zunächst untersucht, ob das Akkulturationsprofil für sich alleine genommen einen Effekt auf die Chance ein Abitur anzustreben hat. Im Vergleich mit Schülerinnen und Schülern mit assimiliertem Akkulturationsprofil (Referenzkategorie) zeigt sich, dass Jugendliche mit einem integrierten Profil $(O R=0,63 ; p<0,05)$ und Jugendliche mit einem separierten Profil $(O R=0,26 ; p<0,001)$ eine geringere Chance haben ein Abitur anzustreben. Für Schülerinnen und Schüler mit moderat 


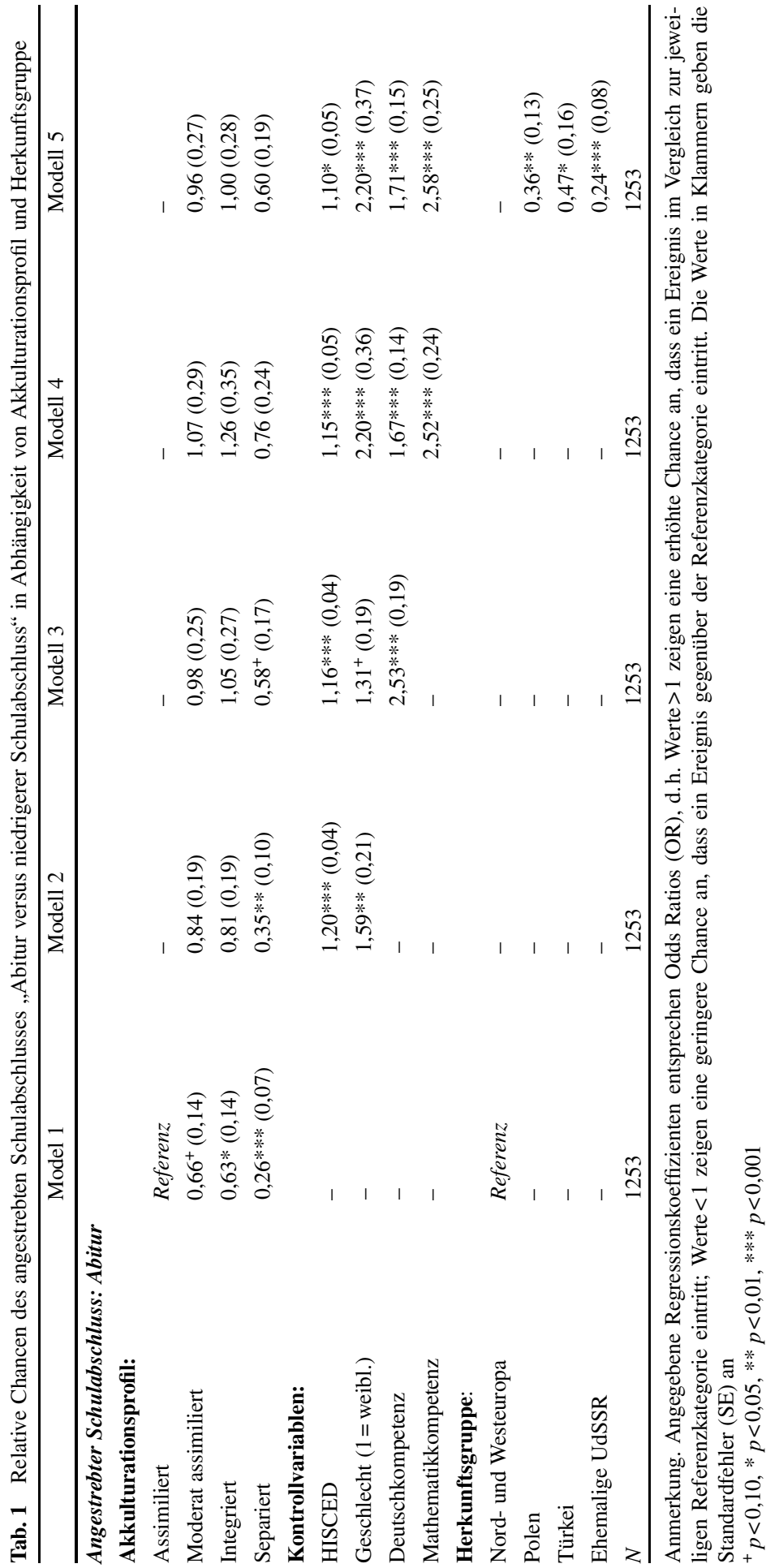




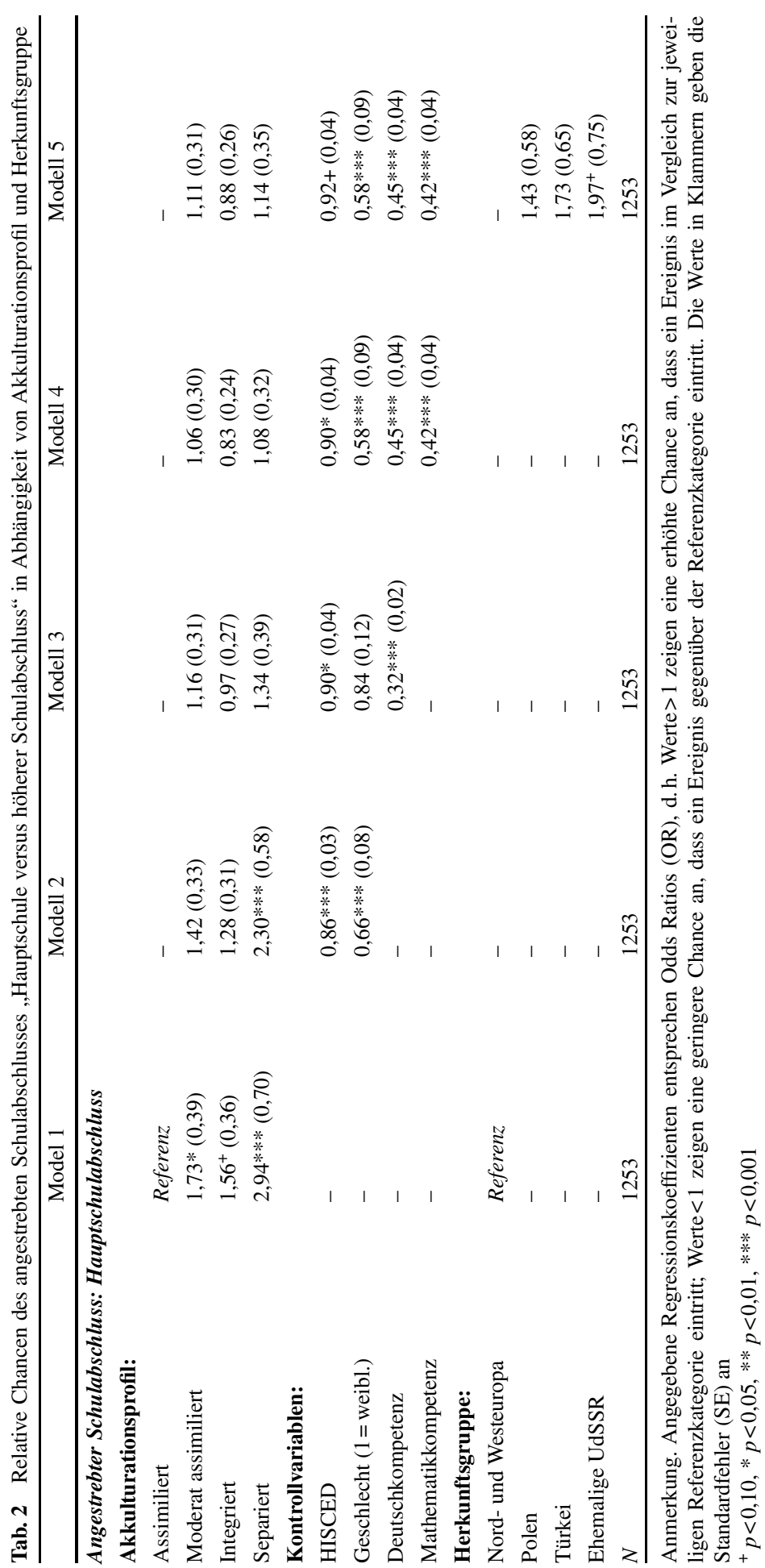


assimiliertem Akkulturationsprofil, zeigt sich im Vergleich zur Referenzgruppe kein signifikanter Unterschied in der Chance ein Abitur anzustreben. In Modell 2 wird geprüft, ob die Effekte des Akkulturationsprofils bei Berücksichtigung des elterlichen Bildungsniveaus (HISCED) und des Geschlechts bestehen bleiben. In Bezug auf den Effekt des Akkulturationsprofils zeigt sich nach Berücksichtigung dieser Kontrollvariablen, dass nur für Schülerinnen und Schüler mit separiertem Profil im Vergleich zu Jugendlichen mit assimiliertem Profil eine signifikant geringere Chance auf das Anstreben eines Abiturs besteht $(O R=0,35, p<0,001)$. Für Jugendliche mit moderat assimiliertem Profil und integriertem Profil unterscheidet sich die Chance ein Abitur anzustreben bei Berücksichtigung von elterlichem Bildungsniveau und Geschlecht nicht signifikant von Jugendlichen mit assimiliertem Profil. Die zusätzliche Berücksichtigung der Deutschkompetenz in Modell 3 führt dazu, dass sich Schülerinnen und Schüler mit separiertem Profil ebenso wie Jugendliche mit moderat assimiliertem und mit integriertem Profil von Jugendlichen mit assimiliertem Profil nicht mehr signifikant in der Chance ein Abitur anzustreben unterscheiden. Bei ergänzender Berücksichtigung der Mathematikkompetenz in Modell 4, steht die Chance ein Abitur anzustreben in keinem signifikanten Zusammenhang mit den Akkulturationsprofilen. Die Aufnahme der Herkunftsgruppen in Modell 5 zeigt, dass zusätzlich ein signifikanter Effekt der Herkunftsgruppe auf die Chance ein Abitur anzustreben besteht: Im Vergleich mit Schülerinnen und Schülern mit nord- und westeuropäischem Migrationshintergrund haben Jugendliche, die eine polnische Herkunft haben $(O R=0,36, p<0,01)$, die eine türkische Herkunft haben $(O R=0,47, p<0,05)$ oder aus der ehemaligen UdSSR stammen $(O R=0,24, p<0,001)$, geringere Chancen ein Abitur anzustreben.

\subsection{Zusammenhang zwischen Akkulturation und der Chance einen Hauptschulabschluss anzustreben}

Die Chance einen Hauptschulabschluss versus einen höheren Schulabschluss (Realschulabschluss und Abitur) anzustreben wurde ebenfalls unter der Voraussetzung unterschiedlicher Akkulturationsprofile (Forschungsfrage 3a) und der Herkunftsgruppe (Forschungsfrage 3b) mittels logistischer Regressionen untersucht (Tab. 2).

In Modell 1 wird zunächst der Effekt der vier ermittelten Akkulturationsprofile auf die Chance einen Hauptschulabschluss anzustreben untersucht. Im Vergleich zu Schülerinnen und Schülern mit assimiliertem Profil (Referenzkategorie) zeigt sich, dass die Chance einen Hauptschulabschluss anzustreben für Schülerinnen und Schüler mit einem moderat assimilierten Profil $(O R=1,73 ; p<0,05)$ wie auch für Schülerinnen und Schüler mit einem separierten Profil $(O R=2,94 ; p<0,001)$ höher ist. Jugendliche mit integriertem Profil unterscheiden sich in der Chance einen Hauptschulabschluss anzustreben nicht signifikant von Jugendlichen mit assimiliertem Profil. Anschließend wird in Modell 2 geprüft, ob die Effekte der Akkulturationsprofile auch bei Berücksichtigung des elterlichen Bildungsniveaus und des Geschlechts bestehen bleiben. Nach Berücksichtigung dieser Kontrollvariablen bleibt die Chance einen Hauptschulabschluss anzustreben für Jugendliche mit separiertem Profil im Vergleich zu Jugendlichen mit assimiliertem Profil weiterhin mehr als doppelt so hoch $(O R=2,30, p<0,001)$, während sich die Chance einen Haupt- 
schulabschluss anzustreben für Jugendliche mit moderat assimiliertem Profil und mit integriertem Profil nicht signifikant von Jugendlichen mit assimiliertem Profil unterscheidet. Die zusätzliche Berücksichtigung der Deutschkompetenz in Modell 3 führt dazu, dass sich Schülerinnen und Schüler mit separiertem Profil ebenso wie Jugendliche mit moderat assimiliertem und mit integriertem Profil von Jugendlichen mit assimiliertem Profil nicht signifikant in der Chance einen Hauptschulabschluss anzustreben unterscheiden. Wird in Modell 4 zusätzlich noch die Mathematikkompetenz berücksichtigt, steht die Chance einen Hauptschulabschluss anzustreben in keinem signifikanten Zusammenhang mit dem Akkulturationsprofil. Die Aufnahme der Herkunftsgruppen in Modell 5 zeigt, dass der ethnische Hintergrund keinen zusätzlichen signifikanten Einfluss auf die Chance einen Hauptschulabschluss anzustreben nimmt: Schülerinnen und Schüler mit polnischem Migrationshintergrund, mit türkischem Migrationshintergrund und aus der ehemaligen UdSSR unterscheiden sich nicht signifikant von Schülerinnen und Schülern nord- und westeuropäischer Herkunft in der Chance einen Hauptschulabschluss anzustreben.

\section{Diskussion}

In dieser Studie ging es darum, den Zusammenhang zwischen der Akkulturation von Schülerinnen und Schülern mit Migrationshintergrund und dem in der Sekundarstufe angestrebten Schulabschluss zu untersuchen.

Während der bisherige Forschungsstand zum Zusammenhang zwischen Akkulturation und Schulerfolg basiert auf Studien, die die Akkulturation von Schülerinnen und Schülern mit Migrationshintergrund nur hinsichtlich einzelner Aspekte der Akkulturation erfassten, führten wir in einem ersten Schritt Latente Profilanalysen (LPA) durch. Latente Profilanalyse eigenen sich um Information unterschiedlicher kontinuierlicher Variablen einzubeziehen und ermöglichen so einen umfassenden Blick und eine empirische Annäherung an die Komplexität des Akkulturationskonstrukts. Ziel war, mithilfe der LPA Akkulturationsprofile zu identifizieren, die sich aus der Kombination unterschiedlicher Aspekte von Akkulturation, nämlich der kulturellen Identität, der Akkulturationseinstellung, dem Akkulturationsverhalten und der linguistischen Akkulturation, ergeben und so für jedes Individuum die Profilzugehörigkeit mit der größten Wahrscheinlichkeit zu ermitteln. Dadurch wurde der Versuch unternommen, die Vielschichtigkeit von Akkulturation zu berücksichtigen wie es beispielsweise gefordert wird von Schwartz et al. (2010) oder Ward (2001). Mit dem Ziel, die von Berry (1997) postulierten Akkulturationsmuster zu replizieren, wurden vier latente Profile identifiziert und inhaltlich interpretiert. Im Einklang mit Berry (1997) ergab die Interpretation ein assimiliertes, ein integriertes und ein separiertes Akkulturationsprofil. Ein Akkulturationsprofil, welches dem Muster der Marginalisierung entsprochen hätte, konnte durch die LPA nicht identifiziert werden. Stattdessen zeigte sich ein Profil, das in der Tendenz dem Akkulturationsmuster Assimilation entsprach, aber aufgrund der nur moderat vom Stichprobenmittelwert abweichenden Tendenzen als moderat assimiliertes Akkulturationsprofil bezeichnet wurde. Dieses Profil vereinte mit knapp 40\% den größten Anteil an Schülerinnen und Schülern auf sich, gefolgt vom integrierten Akkulturationsprofil mit etwa 30- 
prozentigem Anteil. Während das separierte Akkulturationsprofil gut $20 \%$ auf sich vereinte, geht das assimilierte Akkulturationsprofil auf weniger als zehn Prozent der Stichprobe zurück. Insgesamt betrachtet konnten durch die LPA tatsächlich verschiedene Akkulturationsorientierungen nachgewiesen werden. Die von Berry (1997) theoretisch angenommenen Muster der Akkulturation konnten jedoch nur teilweise repliziert werden. Vergleichbar mit den in den USA mittels LPA durchgeführten Akkulturationsstudien mit College-Studierenden konnte auch in unserer Studie keine Akkulturationsorientierung Marginalisierung, die sich daraus ergibt, dass hinsichtlich beider Kulturen eine Orientierung verneint wird, identifiziert werden (Fox et al. 2013; Schwartz und Zamboanga 2008). Einschränkend muss darauf hingewiesen werden, dass sich möglicherweise ein der Marginalisierung entsprechendes Profil, welches sich durch eine unter dem Stichprobenmittelwert liegende Identifikation mit und Einstellung zu sowohl Deutschland als auch dem Herkunftsland, eine niedrige Zustimmung zu den kulturellen Gewohnheiten des Herkunftslandes und einen indifferenten Sprachgebrauch hätte zeigen können, hätte darstellen lassen, wenn nicht a priori das Ergebnis der LPA auf vier Profile beschränkt worden wäre.

Durch die Berücksichtigung unterschiedlicher Akkulturationsaspekte in latenten Akkulturationsprofilen konnten Akkulturationsorientierungen vielschichtiger erfasst werden als in früheren Untersuchungen, in denen nur die ethnische Identität berücksichtigt wurde, oder Akkulturationsorientierungen ausschließlich über die Akkulturationseinstellung, das Akkulturationsverhalten oder den Sprachgebrauch operationalisierten wurden. Zudem erfolgte keine Einteilung in a priori definierte Kategorien, sondern es wurde - aufgrund der latenten Struktur - die Wahrscheinlichkeit einem bestimmten Profil zuzugehören empirisch ermittelt. Dennoch ist einschränkend festzuhalten, dass Akkulturation weit komplexer als durch dieses Verfahren und die darin berücksichtigten Variablen suggeriert wird. Insbesondere wurden Einflüsse des Kontexts, seien es die gesellschaftlichen Akkulturationserwartungen, die Rahmenbedingungen des jeweiligen Schulkontexts oder der spezifischen Situation beispielsweise im Hinblick auf die vorherrschende Zusammensetzung von Mehrund Minderheit und die Proportionen der jeweiligen Gruppen sowie deren wahrgenommene Stellung (Kern et al. 2020; Reinders et al. 2011), nicht betrachtet. Dementsprechend ist nicht auszuschließen, dass die identifizierten Akkulturationsprofile keine unabhängigen, eigenständigen Entscheidungen des Individuums darstellen. So besteht die Möglichkeit, dass für die befragten etwa fünfzehnjährigen Schülerinnen und Schüler die Ausübung kultureller Gewohnheiten und der Sprachgebrauch in der Familie weitgehend von den Eltern vorgegeben werden und nicht notwendigerweise die eigene Akkulturation widerspiegeln. In zukünftigen Studien könnte dieser These durch die Berücksichtigung anderer Variablen, wie beispielsweise dem Sprachgebrauch mit Peers oder der Mediennutzung nachgegangen werden.

Ausgehend von den empirisch ermittelten Akkulturationsprofilen untersuchten wir in einem zweiten Schritt den Zusammenhang zwischen Akkulturation und angestrebtem Schulabschluss. In den wenigen für den deutschsprachigen Kontext vorliegenden Studien zum Zusammenhang zwischen der Akkulturation und der schulischen Adaptation von Schülerinnen und Schülern mit Migrationshintergrund wurde Schulerfolg bislang überwiegend operationalisiert über die Lesekompetenz (Edele et al. 2013; Hannover et al. 2013; Schotte et al. 2018). Um diesen Forschungsstand 
perspektivisch zu erweitern, entschieden wir uns in dieser Studie als Kriterium für Schulerfolg für den von Schülerinnen und Schülern angestrebten Schulabschluss, welchen wir operationalisierten über den in der neunten Klasse besuchten Bildungszweig. Zwar determiniert die in der Sekundarstufe besuchte Schulform den Schulabschluss nicht, jedoch prädiziert die Schulform für drei von vier Schülerinnen und Schülern den im Erwachsenenalter tatsächlich erreichten Bildungsabschluss (Glaesser und Lauterbach 2006). Eine Mehrheit verlässt demnach die Schule mit dem Abschluss der Schulform, die sie im Alter von 15 Jahren besucht haben. Untersucht wurde die Chance ein Abitur versus einen niedrigeren Schulabschluss anzustreben (Fragestellung 2a) und die Chance einen Hauptschulabschluss versus einen höheren Schulabschluss anzustreben (Fragestellung 3a) in Abhängigkeit eines moderat assimilierten, integrierten oder separierten Akkulturationsprofilen im Vergleich zu einem assimilierten Akkulturationsprofil. In Anlehnung an Studien, die einen Zusammenhang zwischen der in der Sekundarstufe besuchten Schulform und der Herkunftsgruppe zeigen konnten (Kristen 2002; Segeritz et al. 2010) wurde zusätzlich zur Akkulturation der Effekt der Herkunftsgruppe der Schülerinnen und Schüler untersucht (Fragestellungen 2b und 3b). Die Ergebnisse der logistischen Regression zur Vorhersage der Chance ein Abitur anstelle eines niedrigeren Schulabschlusses anzustreben ergaben, dass die relative Chance auf ein Abitur für Schülerinnen und Schüler mit integriertem und separiertem Akkulturationsprofil geringer ist als für Schülerinnen und Schüler mit assimiliertem Akkulturationsprofil. Für das integrierte Akkulturationsprofil ist dieser Effekt jedoch nicht mehr signifikant, wenn das Geschlecht sowie das elterliche Bildungsniveau berücksichtigt werden. Wird zusätzlich für die Kompetenz in Deutsch (und Mathematik) kontrolliert, kann die geringere Chance auf ein Abitur für Schülerinnen und Schüler mit separiertem Akkulturationsprofil nicht mehr gegen den Zufall abgesichert werden. Die Ergebnisse der logistischen Regression zur Vorhersage der Chance einen Hauptschulabschluss anstelle eines höheren Schulabschlusses anzustreben ergab, dass die relative Chance auf einen Hauptschulabschluss deutlich höher ist für Schülerinnen und Schüler mit einem moderat assimilierten und separierten Akkulturationsprofil als für Schülerinnen und Schüler mit assimiliertem Akkulturationsprofil. Wurden das Geschlecht und das elterliche Bildungsniveau berücksichtigt, war die Chance auf einen Hauptschulabschluss für Schülerinnen und Schüler mit separiertem Akkulturationsprofil weiterhin deutlich höher als für Schülerinnen und Schüler mit assimiliertem Akkulturationsprofil, nicht aber für Schülerinnen und Schüler mit moderat assimiliertem Akkulturationsprofil. Bei zusätzlicher Kontrolle der Kompetenz in Deutsch (und Mathematik) zeigten sich keine Effekte, die auf die Akkulturationsprofile zurückzuführen sind. Wurde zusätzlich die Herkunftsgruppe berücksichtigt, zeigte sich, dass die Chance geringer ist für Schülerinnen und Schüler, deren Familien aus Polen, der Türkei oder der ehemaligen UdSSR stammen, ein Abitur anzustreben als für Schülerinnen und Schüler mit einem nord- bzw. westeuropäischen Migrationshintergrund, auch wenn das elterliche Bildungsniveau, das Geschlecht und die Kompetenzen in Deutsch und Mathematik berücksichtigt wurden. Für das Anstreben eines Hauptschulabschlusses ließen sich in der Tendenz höhere Chancen der Herkunftsgruppen Polen, Türkei und ehemalige UdSSR zeigen, die jedoch nicht signifikant waren. 
Zusammengefasst zeigt sich, dass etwa $22 \%$ der Schülerinnen und Schüler mit Migrationshintergrund einem separierten Akkulturationsprofil zugeordnet werden können und diese Schülerinnen und Schüler signifikant niedriger ein Abitur und signifikant höher einen Hauptschulabschluss anstreben. Dabei ist zum einen das elterliche Bildungsniveau zentral und zum anderen stellt die Deutschkompetenz einen entscheidenden Faktor dar. Die Verflochtenheit von Migrationshintergrund, dem Bildungsstand der Eltern und der Deutschkompetenz, wie sie vor allem im Hinblick auf das Anstreben eines niedrigen Schulabschlusses zu erwarten ist, ist schwer aufzulösen und reduziert die Rolle der Akkulturationsorientierung. Dennoch erweitert unsere Studie den Forschungsstand zum Zusammenhang zwischen ethnischer Herkunft und besuchter Schulform, indem sich Zusammenhänge unterschiedlicher Akkulturationsprofile mit dem angestrebten Schulabschluss aufzeigen lassen, die jedoch unter Einbezug weiterer Variablen an Bedeutung verlieren. Außerdem sind unsere Ergebnisse im Einklang mit Studien, deren Befunde anstelle der besonderen Adaptation der Integration auf den Vorteil der kulturellen Assimilation, d.h. einer starken Orientierung an der deutschen Kultur bei Schülerinnen und Schülern verweisen (Edele et al. 2013; Makarova und Birman 2015; Schotte et al. 2018). Weiter verweisen unsere Ergebnisse auf einen Nachteil kultureller Separation, die sich durch eine schwache Orientierung an der Kultur des Aufnahmelandes bei gleichzeitig starker Orientierung an der Kultur des Herkunftslandes auszeichnet. Nach der Berücksichtigung der Deutschkompetenz, kann der Zusammenhang zwischen Akkulturation und separiertem Akkulturationsprofil wie auch bei Edele et al. (2013) nicht mehr gegen den Zufall abgesichert werden. Durch die Berücksichtigung unterschiedlicher Aspekte von Akkulturation in unserer LPA, kann dieses Ergebnis auf den Sprachgebrauch in der Familie zurückgeführt werden. Wie die Profile der LPA zeigen, wird in den Familien von Schülerinnen und Schülern mit separiertem Akkulturationsprofil überwiegend eine andere Sprache als Deutsch gesprochen. Dies weist einerseits, mit Blick auf das Individuum, auf die zentrale Rolle der Deutschkompetenz für Schulerfolg hin. Andererseits, mit Blick auf die gesellschaftlichen Erwartungen und Rahmenbedingungen gerade auch im schulischen Kontext, weist dieser Befund auf den monolingualen Habitus der Schule hin (Gogolin 1994). Die Annahme, dass Schülerinnen und Schüler mit einer schwachen Orientierung an beiden Kulturen Nachteile im Hinblick auf den angestrebten Schulabschluss haben, konnten unsere Ergebnisse nicht zeigen, da sich kein der Marginalisierung entsprechendes Akkulturationsprofil darstellte. Darüber hinaus gehend weisen unsere Ergebnisse darauf hin, dass die häufig gezeigte besondere Bildungsungleichheit von Schülerinnen und Schülern mit türkischem Migrationshintergrund und für aus der ehemaligen UdSSR stammende Schülerinnen und Schüler, bei Berücksichtigung der Akkulturation und relevanter Hintergrundvariablen ebenso für Schülerinnen und Schüler mit polnischem Migrationshintergrund - zumindest im Hinblick auf das Anstreben eines Abiturs, existiert. Der Vorteil von Schülerinnen und Schülern mit nord- oder westeuropäischem Migrationshintergrund kann in Anlehnung an akkulturationstheoretische Überlegungen auf die kulturelle Nähe zu Deutschland zurückgeführt werden (Berry 1997).

Neben den bereits genannten Limitationen der Studie liegt eine weitere Einschränkung in der Auswahl des Samples vor. Aufgrund der Begrenzung der Herkunftsgruppen auf die vier größten Gruppen im NEPS-Datensatz sind keine Rückschlüsse auf 
die in Deutschland heterogene Gruppe der Schülerinnen und Schüler mit Migrationshintergrund möglich (siehe Moffitt und Juang 2019). Dies trifft insbesondere auf die in jüngerer Vergangenheit nach Deutschland Migrierten zu. Außerdem sei an dieser Stelle betont, dass unsere Befunde keine verallgemeinernden Folgerungen über einzelne Individuen einer kulturellen Gruppe oder einer bestimmten Akkulturationsorientierung ermöglichen. Im NEPS wird Schülerinnen und Schülern bis in die dritte Generation ein Migrationsstatus zugewiesen (Kristen et al., 2016). Dies entspricht nicht der in der empirischen Bildungsforschung aktuell üblichen Definition eines Migrationshintergrundes (z.B. OECD 2019), nach der ein Migrationshintergrund nur bis in die zweite Generation, sofern beide Elternteile immigriert sind, zugewiesen wird. Dennoch wurden in unsere Analysen alle Schülerinnen und Schüler der genannten Herkunftsgruppen unabhängig vom Generationenstatus berücksichtigt, wenn sie entsprechende Angaben zu ihren kulturellen Orientierungen gemacht hatten. Einschränkend muss weiter vermerkt werden, dass aufgrund des QuerschnittDesigns der Studie lediglich Zusammenhänge zwischen den ermittelten Akkulturationsprofilen und der besuchten Schulform zum Zeitpunkt der Erhebung analysiert wurden und damit keine Rückschlüsse auf kausale Zusammenhänge möglich sind. Die bei den Schülerinnen und Schülern in der 9. Klasse gemessenen Einstellungen und das gemessene Verhalten haben somit keinen Einfluss auf die Wahl des Bildungsgangs, welcher ohnehin schon viel früher durch elterliche und lehrerseitige Entscheidungen, möglicherweise aufgrund der damaligen Deutschkompetenz, getroffen wurde. Zudem bleibt in unserer Studie offen, inwiefern der über den besuchten Bildungszweig ermittelte - voraussichtlich zu erreichende - Schulabschluss dem intendierten, im Sinne der Bildungsaspiration explizit gewünschten Schulabschluss entspricht. Akkulturation ist ein Prozess, der nicht unabhängig von distalen, gesellschaftlichen Einflüssen ist und für den proximale Faktoren, wie z. B. Transmissionsprozesse der Eltern und Peers, entscheidend sind. Wünschenswert wäre daher, dass dies in zukünftigen Studien in entsprechend komplexen Modellen Berücksichtigung findet und im Längsschnitt untersucht wird. Insgesamt decken sich unsere Ergebnisse mit Befunden, die zeigen, dass in erster Linie Kompetenzunterschiede für die schulische Positionierung von Schülerinnen und Schülern mit Migrationshintergrund ursächlich sind (Siegert und Olszenka 2016). In Anbetracht der Tatsache, dass in der vorliegenden Stichprobe für jede fünfte Schülerin und jeden fünften Schüler mit Migrationshintergrund, nämlich diejenigen mit separiertem Akkulturationsprofil, gezeigt werden kann, dass Nachteile im Hinblick auf den angestrebten Schulabschluss auf schwache deutschsprachliche Kompetenzen zurückgeführt werden können, muss daraus konkret die Schlussfolgerung gezogen werden, dass insbesondere die Sprachkompetenz ob ihrer zentralen Bedeutung für den Bildungserfolg von Schülerinnen und Schülern mit Migrationshintergrund eine Priorität im schulischen Handeln darstellen sollte. Auch Berry (2013) betont die Bedeutung der Rolle der Sprache im multikulturellen Kontext. Neben direkter Maßnahmen der Sprachförderung im Unterricht, nicht erst in der Sekundarstufe, sollte auch über indirekte Möglichkeiten wie beispielsweise gezielte Peerkontakte nachgedacht werden, die nebenbei durch das Gefühl sozialer Eingebundenheit zu positiven Veränderungen in der Zugehörigkeit und dem Verbundenheitsgefühl führen und letztlich eine stärkere Orientierung der Schülerinnen und Schüler mit Migrationshintergrund an der Auf- 
nahmekultur nach sich ziehen könnte. Um letztlich klären zu können, ob es sich bei der Konzentration von Akkulturationsprofilen auf bestimmten Bildungszweigen möglicherweise um Sozialisations- oder Selektionseffekte handelt, bedarf es einer Analyse im Längsschnitt.

Funding Acknowledgment Gefördert durch die Deutsche Forschungsgemeinschaft (DFG) - Projektnummer: LI 3067-1/1.

Funding Open Access funding enabled and organized by Projekt DEAL.

Open Access Dieser Artikel wird unter der Creative Commons Namensnennung 4.0 International Lizenz veröffentlicht, welche die Nutzung, Vervielfältigung, Bearbeitung, Verbreitung und Wiedergabe in jeglichem Medium und Format erlaubt, sofern Sie den/die ursprünglichen Autor(en) und die Quelle ordnungsgemäß nennen, einen Link zur Creative Commons Lizenz beifügen und angeben, ob Änderungen vorgenommen wurden.

Die in diesem Artikel enthaltenen Bilder und sonstiges Drittmaterial unterliegen ebenfalls der genannten Creative Commons Lizenz, sofern sich aus der Abbildungslegende nichts anderes ergibt. Sofern das betreffende Material nicht unter der genannten Creative Commons Lizenz steht und die betreffende Handlung nicht nach gesetzlichen Vorschriften erlaubt ist, ist für die oben aufgeführten Weiterverwendungen des Materials die Einwilligung des jeweiligen Rechteinhabers einzuholen.

Weitere Details zur Lizenz entnehmen Sie bitte der Lizenzinformation auf http://creativecommons.org/ licenses/by/4.0/deed.de.

\section{Literatur}

Arends-Tóth, J., \& van de Vijver, F. J. R. (2004). Domains and dimensions in acculturation: implicit theories of Turkish-Dutch. International Journal of Intercultural Relations, 28(1), 19-35. https://doi. org/10.1016/j.ijintrel.2003.09.001.

Arends-Tóth, J., \& van de Vijver, F. J.R. (2006). Issues in the conceptualization and assessment of acculturation. In H. Bornstein \& L. R. Cote (Hrsg.), Acculturation and parent-child relationships. Measurement and development (S. 33-62). New Jersey: Lawrence Erlbaum.

Autorengruppe Bildungsberichterstattung (2018). Bildung in Deutschland 2018. Ein indikatorengestützter Bericht mit einer Analyse zu Wirkungen und Erträgen von Bildung (1. Aufl.). Bielefeld: wbv.

Baier, D., \& Pfeiffer, C. (2014). Wer sind die Aufsteiger, wer die Absteiger im deutschen Bildungssystem? Eine Analyse auf Basis einer deutschlandweit repräsentativen Befragung und besonderer Berücksichtigung des Geschlechts. In D. Baier, T. Mößle \& C. Pfeiffer (Hrsg.), Die Krise der Jungen: Phänomenbeschreibung und Erklärungsansätze (S. 29-60). Baden-Baden: Nomos. https://doi.org/10.5771/ 9783845258560_29.

Baumert, J., \& Schümer, G. (2001). Familiäre Lebensverhältnisse, Bildungsbeteiligung und Kompetenzerwerb. In J. Baumert, C. Artelt, E. Klieme, M. Neubrand, M. Prenzel, U. Schiefele, W. Schneider, K.-J. Tillmann \& M. Weiß (Hrsg.), PISA 2000: Basiskompetenzen von Schülerinnen und Schülern im internationalen Vergleich (S. 323-407). Opladen: Leske + Budrich.

Berry, J. W. (1997). Immigration, acculturation, and adaptation. Applied Psychology, 46(1), 5-34. https:// doi.org/10.1111/j.1464-0597.1997.tb01087.x.

Berry, J.W. (2001). A psychology of immigration. Journal of Social Issues, 57(3), 615-631. https://doi. org/10.1111/0022-4537.00231.

Berry, J. W. (2005). Acculturation: living successfully in two cultures. International Journal of Intercultural Relations, 29(6), 697-712. https://doi.org/10.1016/j.ijintrel.2005.07.013.

Berry, J. W. (2006). Mutual attitudes among immigrants and ethnocultural groups in Canada. International Journal of Intercultural Relations, 30(6), 719-734. https://doi.org/10.1016/j.ijintrel.2006.06.004.

Berry, J. W. (2013). Intercultural relations in plural societies: research derived from multiculturalism policy. Acta de Investigación Psicológica, 3(2), 1122-1135. https://doi.org/10.1016/S2007-4719(13)709566. 
Blossfeld, H.-P., Roßbach, H.-G., \& von Maurice, J. (Hrsg.). (2011). Education as a lifelong process: The German National Educational Panel Study (NEPS) (Zeitschrift für Erziehungswissenschaft: Sonderheft 14). Wiesbaden: Springer VS.

Dollmann, J. (2016). Der Übergang von der Primar- in die Sekundarstufe. In C. Diehl, C. Hunkler \& C. Kristen (Hrsg.), Ethnische Ungleichheiten im Bildungsverlauf (S. 517-542). Wiesbaden: Springer VS. https://doi.org/10.1007/978-3-658-04322-3_12

Edele, A., Stanat, P., Radmann, S., \& Segeritz, M. (2013). Kulturelle Identität und Lesekompetenz von Jugendlichen aus zugewanderten Familien. Zeitschrift für Pädagogik, 59, 84-110. Beiheft.

Fox, R. S., Merz, E. L., Solórzano, M. T., \& Roesch, S.C. (2013). Further examining Berry's model: the applicability of latent profile analysis to acculturation. Measurement and Evaluation in Counseling and Development, 46(4), 270-288. https://doi.org/10.1177/0748175613497036.

Glaesser, J., \& Lauterbach, W. (2006). Prädiziert die Schulform auch den Schulabschluss? Oder: Auf- und Absteiger aus schulischen Laufbahnen. In K.-S. Rehberg (Hrsg.), Soziale Ungleichheit, kulturelle Unterschiede. Verhandlungen des 32. Kongresses der Deutschen Gesellschaft für Soziologie in München (S. 1741-1753). Frankfurt a. M.: Campus. CD-ROM.

Gogolin, I. (1994). Der monolinguale Habitus der multilingualen Schule. Münster: Waxmann.

Graves, T. D. (1967). Psychological acculturation in a tri-ethnic community. Southwestern Journal of Anthropology, 23(4), 337-350.

Hachfeld, A., \& Syring, M. (2020). Stichwort: Überzeugungen von Lehrkräften im Kontext migrationsbezogener Heterogenität. Zeitschrift für Erziehungswissenschaft, 23(4), 659-684. https://doi.org/10. 1007/s11618-020-00957-7.

Hannover, B., Morf, C.C., Neuhaus, J., Rau, M., Wolfgramm, C., \& Zander-Musić, L. (2013). How immigrant adolescents' self-views in school and family context relate to academic success in Germany. Journal of Applied Social Psychology, 43(1), 175-189. https://doi.org/10.1111/j.1559-1816.2012. 00991.x.

Kenji, H., \& D’Andrea, D. (1992). Some properties of bilingual maintenance and loss in Mexican background high-school students. Applied Linguistics, 13(1), 72-99. https://doi.org/10.1093/applin/13.1. 72.

Kern, M. R., Heinz, A., \& Willems, H.E. (2020). School-class co-ethnic and immigrant density and current smoking among immigrant adolescents. International Journal of Environmental Research and Public Health, 17(2), 598. https://doi.org/10.3390/ijerph17020598.

Kristen, C. (2002). Hauptschule, Realschule oder Gymnasium? KZfSS Kölner Zeitschrift für Soziologie und Sozialpsychologie, 54(3), 534-552. https://doi.org/10.1007/s11577-002-0073-2.

Kristen, C., Olczyk, M., \& Will, G. (2016). Identifying immigrants and their descendants in the National Educational Panel Study. In H. P. Blossfeld, J. von Maurice, M. Bayer \& J. Skopek (Hrsg.), Methodological issues of longitudinal surveys. Wiesbaden: Springer VS. https://doi.org/10.1007/978-3-65811994-2_12.

Lenz, S., Holtmann, M., Rjosk, C., \& Stanat, P. (2019). Soziokulturelle Segregation an weiterführenden Schulen - Analysen zur Rolle der Gliederung des deutschen Schulsystems und schulstruktureller Reformmaßnahmen. Zeitschrift für Erziehungswissenschaft, 22(6), 1333-1358. https://doi.org/10.1007/ s11618-019-00913-0.

Makarova, E., \& Birman, D. (2015). Cultural transition and academic achievement of students from ethnic minority backgrounds: a content analysis of empirical research on acculturation. Educational Research, 57(3), 305-330. https://doi.org/10.1080/00131881.2015.1058099.

Moffitt, U., \& Juang, L.P. (2019). Who is "German" and who is a "migrant?" Constructing otherness in education and psychology research. European Educational Research Journal, 18(6), 656-674. https:// doi.org/10.1177/1474904119827459.

Müller, A. G., \& Stanat, P. (2006). Schulischer Erfolg von Schülerinnen und Schülern mit Migrationshintergrund. Analysen zur Situation von Zuwanderern aus der ehemaligen Sowjetunion und aus der Türkei. In J. Baumert, P. Stanat \& R. Watermann (Hrsg.), Herkunftsbedingte Disparitäten im Bildungswesen. Differenzielle Bildungsprozesse und Probleme der Verteilungsgerechtigkeit; vertiefende Analysen im Rahmen von PISA 2000 (S. 221-255). Wiesbaden: VS.

Nieri, T., Lee, C., Kulis, S., \& Marsiglia, F.F. (2011). Acculturation among Mexican-heritage preadolescents: a latent class analysis. Social Science Research, 40(4), 1236-1248. https://doi.org/10.1016/j. ssresearch.2011.02.005.

Nylund-Gibson, K., \& Choi, A. Y. (2018). Ten frequently asked questions about latent class analysis. Translational Issues in Psychological Science, 4(4), 440-461. https://doi.org/10.1037/tps0000176.

OECD (1999). Classifying educational programmes. Manual for ISCED-97 implementation in OECD countries-1999 Edition. Paris: OECD. 
Olczyk, M., Seuring, J., Will, G., \& Zinn, S. (2016). Migranten und ihre Nachkommen im deutschen Bildungssystem: Ein aktueller Überblick. In C. Diehl, C. Hunkler \& C. Kristen (Hrsg.), Ethnische Ungleichheiten im Bildungsverlauf (S. 33-70). Wiesbaden: Springer VS. https://doi.org/10.1007/978-3658-04322-3_2

OECD (2019). PISA 2018 Results (Volume II): Where All Students Can Succeed, PISA. Paris: OECD Publishing. https://doi.org/10.1787/b5fd1b8f-en

Phinney, J.S. (1992). The multigroup ethnic identity measure: a new scale for use with diverse groups. Journal of Adolescent Research, 7(2), 156-176. https://doi.org/10.1177/074355489272003.

Pohl, S., \& Carstensen, C. (2012). NEPS technical report-scaling the data of the competence tests (NEPS working paper: Nr. 14). Bamberg: NEPS.

Redfield, R., Linton, R., \& Herskovits, M. J. (1936). Memorandum for the study of acculturation. American Anthropologist, 38(1), 149-152.

Reinders, H., Gniewosz, B., Gresser, A., \& Schnurr, S. (2011). Erfassung interkultureller Kompetenzen bei Kindern und Jugendlichen. Das Würzburger Interkulturelle Kompetenz-Inventar (WIKI-KJ). Diskurs Kindheits- und Jugendforschung, 4, 429-452.

Rudmin, F. W. (2003). Critical history of the acculturation psychology of assimilation, separation, integration, and marginalization. Review of General Psychology, 7(1), 3-37. https://doi.org/10.1037/10892680.7.1.3.

Schnell, R. (1990). Dimensionen ethnischer Identität. In H. Esser \& J. Friedrichs (Hrsg.), Generation und Identität (S. 43-72). Wiesbaden: Vieweg+Teubner Verlag. https://doi.org/10.1007/978-3-322-917775_3.

Schotte, K., Stanat, P., \& Edele, A. (2018). Is integration always most adaptive? The role of cultural identity in academic achievement and in psychological adaptation of immigrant students in Germany. Journal of Youth and Adolescence, 47, 16-37. https://doi.org/10.1007/s10964-017-0737-x.

Schwartz, S. J., \& Zamboanga, B. L. (2008). Testing Berry's model of acculturation: a confirmatory latent class approach. Cultural Diversity and Ethnic Minority Psychology, 14(4), 275-285. https://doi.org/ $10.1037 / \mathrm{a} 0012818$.

Schwartz, S. J., Unger, J. B., Zamboanga, B. L., \& Szapocznik, J. (2010). Rethinking the concept of acculturation: implications for theory and research. American Psychologist, 65(4), 237-251. https://doi. org/10.1037/a0019330.

Segeritz, M., Walter, O., \& Stanat, P. (2010). Muster des schulischen Erfolgs von jugendlichen Migranten in Deutschland: Evidenz für segmentierte Assimilation? KZfSS Kölner Zeitschrift für Soziologie und Sozialpsychologie, 62(1), 113-138. https://doi.org/10.1007/s11577-010-0094-1.

Siegert, M., \& Olszenka, N. (2016). Ethnische Ungleichheit in der Sekundarstufe I. In C. Diehl, C. Hunkler \& C. Kristen (Hrsg.), Ethnische Ungleichheiten im Bildungsverlauf (S. 543-595). Wiesbaden: Springer. https://doi.org/10.1007/978-3-658-04322-3_13.

Solga, H., \& Wagner, S. (2010). Die Zurückgelassenen - die soziale Verarmung der Lernumwelt von Hauptschülerinnen und Hauptschülern. In R. Becker \& W. Lauterbach (Hrsg.), Bildung als Privileg (S. 191-219). Wiesbaden: VS. https://doi.org/10.1007/978-3-531-92484-7_7.

Ward, C. (2001). The ABCs of acculturation. In D. Matsumoto (Hrsg.), Handbook of culture and psycho$\log y$ (S. 411-445). Oxford: University Press.

Weis, M., Müller, K., Mang, J., Heine, J.-H., Mahler, N., \& Reiss, K. (2019). Soziale Herkunft, Zuwanderungshintergrund und Lesekompetenz. In K. Reiss, M. Weis, E. Klieme \& O. Köller (Hrsg.), PISA 2018. Grundbildung im internationalen Vergleich (S. 129-162). Münster: Waxmann. https://doi.org/ $10.31244 / 9783830991007$.

Wendt, H., \& Schwippert, K. (2017). Lesekompetenzen von Schülerinnen und Schülern mit und ohne Migrationshintergrund. In A. Hussmann, H. Wendt, W. Bos, A. Bremerich-Vos, D. Kasper, E.-M. Lankes, N. McElvany, T. C. Stubbe \& R. Valtin (Hrsg.), IGLU 2016: Lesekompetenzen von Grundschulkindern in Deutschland im internationalen Vergleich (S. 219-234). Münster: Waxmann. 